\title{
Electrostatic ring as the central machine of the Frankfurt Ion Storage Experiments
}

\author{
C. P. Welsch* and J. Ullrich \\ Max-Planck Institut für Kernpyhsik, Heidelberg, Germany \\ C. Gläßner, K.-U. Kühnel, and A. Schempp \\ IAP, Goethe University, Frankfurt, Germany \\ H. Schmidt-Böcking and R. Dörner \\ IKF, Goethe University, Frankfurt Germany \\ (Received 19 April 2004; published 13 August 2004)
}

\begin{abstract}
A small electrostatic storage ring is the central machine of the Frankfurt Ion Storage Experiments (FIRE) which will be built at the new Stern-Gerlach Center of Frankfurt University. As a true multiuser, multipurpose facility with ion energies up to $50 \mathrm{keV}$, it will allow new methods to analyze complex many-particle systems from atoms to very large biomolecules. With envisaged storage times of some seconds and beam emittances in the order of a few $\mathrm{mm}$ mrad, measurements with up to 6 orders of magnitude better resolutions as compared to single-pass experiments become possible. In comparison to earlier designs, the ring lattice was modified in many details: Problems in earlier designs were related to, e.g., the detection of light particles and highly charged ions with different charge states. Therefore, the deflectors were redesigned completely, allowing a more flexible positioning of the diagnostics. Here, after an introduction to the concept of electrostatic machines, an overview of the planned FIRE is given and the ring lattice and elements are described in detail.
\end{abstract}

DOI: 10.1103/PhysRevSTAB.7.080101

PACS numbers: $29.17 .+\mathrm{w}$

\section{INTRODUCTION}

Existing electrostatic storage rings [1,2] have proven to be a valuable tool for molecular and atomic physics in the low-energy regime. With the primary goal to analyze biomolecules and negative ions, many interesting new results could be achieved [3-5].

The main difference between magnetic rings and their electrostatic counterparts can be seen by comparing the electrostatic and magnetic rigidity:

$$
\begin{gathered}
\rho E=\frac{2 E_{\mathrm{kin}}}{q}, \\
\rho B=\frac{1}{q} \sqrt{2 m E_{\mathrm{kin}}} .
\end{gathered}
$$

The bending effect in the electrostatic case (1) depends only on the particle's kinetic energy $E_{\text {kin }}$ and charge state $q$ and is independent of the ion's mass $m$, while the rigidity is mass dependent in the magnetic case.

It should be pointed out that the necessary electric field to bend a particle a certain radius $\rho$ scales linear with the kinetic energy and thus limits electrostatic elements to relatively low beam energies, while the square root law (2) in magnetic bending elements enables the storage of particles up to highest energies.

At the new Stern-Gerlach Center of Frankfurt University a small machine for ion energies up to

\footnotetext{
*carsten.welsch@mpi-hd.mpg.de
} the structure and dynamics of many-particle systems from atoms to complex organic biomolecules. It will be possible to prepare the particle beams of interest in novel and unique ways.

For the first time, a reaction microscope [6] will be integrated in a low-energy storage ring, allowing the Frankfurt Ion Storage Experiments (FIRE) the analysis of many-particle fragmentation processes of atoms and molecules with unrivaled resolution and completeness. In contrast to experiments with traps, an electrostatic storage ring has the advantage of being able to record the momenta of all neutral fragments.

In such a machine, molecules can be fragmented and detected by state-of-the-art imaging techniques with high momentum resolution. In a final step, the measurement of dynamic correlations in the 10 attosecond regime can be envisioned. In traps, where ions are controlled by nonlinear electric and magnetic fields, such precision measurements are hardly possible.

\section{EXPERIMENTAL GOALS}

In the ring of FIRE, only electrostatic elements will be used. Because of the mass independence of the electrostatic rigidity, a wide range of atomic and molecular ions can be stored in the machine. This is of particular benefit during the setup of the storage ring, since an intense test beam can be used in the beginning and then injection can be changed to the probably much weaker beam of interest. 
While electrostatic rings concentrated on experiments with continuous beams so far [7,8], a bunched structure in the machine is a central requirement for the planned collision experiments at FIRE. During the past 15 years the development of COLTRIMS [6] and multifragment imaging techniques [9] was one of the central research fields at IKF, Frankfurt. Based on this experience, an inring reaction microscope could greatly improve present experimental resolutions and allow kinematically complete analysis of fragments resulting from collision of the stored beam and a cold target, focusing on the studies of complex organic matter. Furthermore, the circulating beam can be merged with, e.g., electron or laser beams and set to a variety of interesting quantum mechanical states allowing completely novel experiments.

Future facilities like the cryogenic storage ring at the Max-Planck Institut, Heidelberg [10], the DESIREE project in Stockholm [11], or the proposed FLAIR facility at GSI [12] will use electrostatic rings as a central machine and the experience from the ring of FIRE will be extremely beneficial for these projects.

\section{RING LATTICE}

The machine consists of two different types of bending elements - parallel plate and cylindrical deflectors - and quadrupole doublets for transverse focusing of the beam. A double symmetric layout was chosen to keep the lattice as simple as possible and to be able to use identical design for the elements. As can be seen in Figs. 1 and 2, experimental requirements had a great influence on the design of the vacuum chambers. Easy access to all the elements is important for a machine, where a wide range of fields will

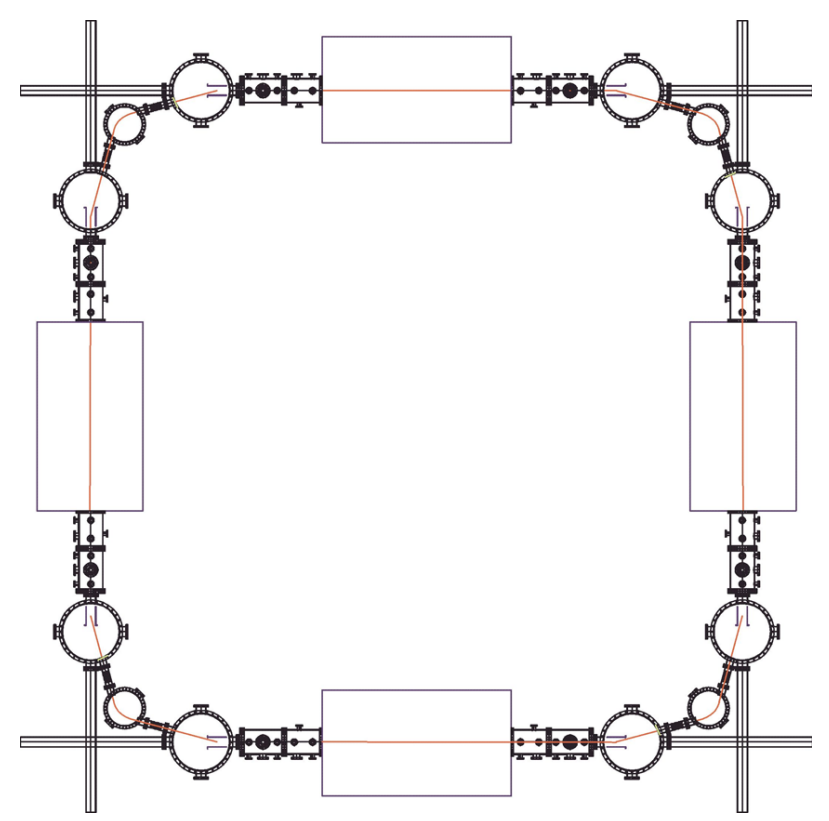

FIG. 1. (Color) Overview of the ring of FIRE. be covered and the included diagnostic elements will have to change depending on the experiment carried out.

Therefore, pillbox-type cavities were used allowing easy adjustment of the optical elements and giving enough room for additional detectors.

The size of machine of about $7 \mathrm{~m} \times 7 \mathrm{~m}$ is dominated by the experimental section, where a length of $2 \mathrm{~m}$ is foreseen for in-ring experiments like the reaction microscope and an electron target. By splitting up the $90^{\circ}$ bends in the corner sections into two $15^{\circ}$ parallel plate deflectors and one $60^{\circ}$ cylinder deflector, single turn injection can be done along one of the straight sections with one of the deflectors being switched off in the beginning. After energy separation in the injection channel, the beam is directly focused into the machine.

Once half of the ring's circumference is filled with ions, the parallel plate deflector is turned on and the beam can be stored. The revolution time of the lightest ions that will be stored, protons, is roughly $10 \mu \mathrm{s}$ and thus the ramping time of the pulsed deflector used for injection of $300 \mathrm{nsec}$ is reasonably short.

In addition, the separation of the bending elements in the corner sections allows easy monitoring of neutral particles created along the straight sections and also the injection of merged beams.

A bunched beam structure is realized by an rf driven drift tube where voltages $<100 \mathrm{~V}$ will be applied to counteract the natural longitudinal debunching effects.

Depending on the experimental needs, the machine can be set to different working modes. Four stable working points exist in the basic mode where the beam is wide in the experimental sections; see Fig. 3.

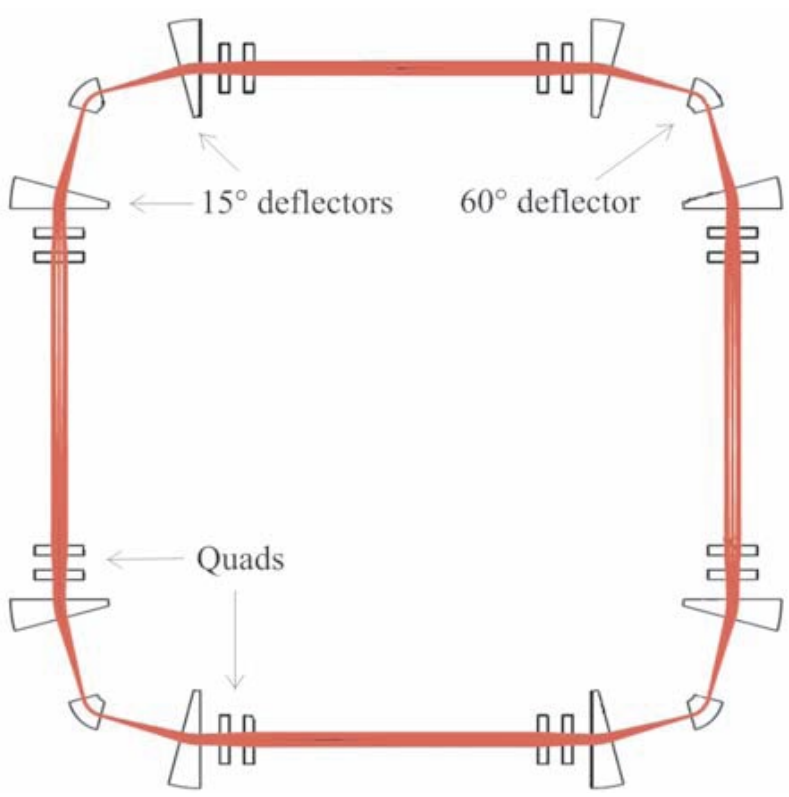

FIG. 2. (Color) Beam envelope calculated with COSY infinity. Transverse dimensions are multiplied by a factor of 5 . 
I

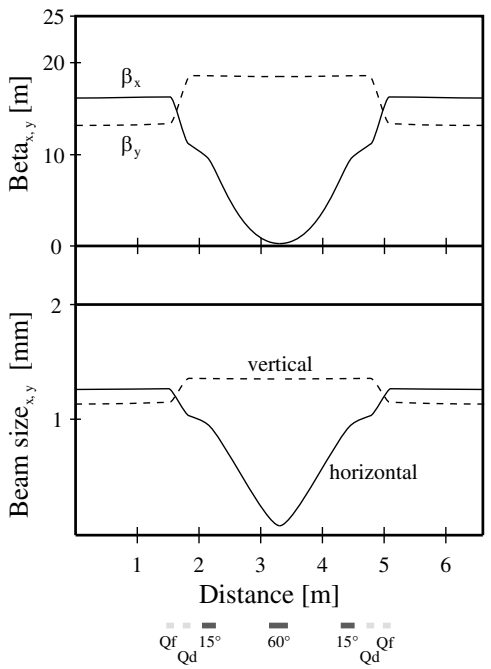

III

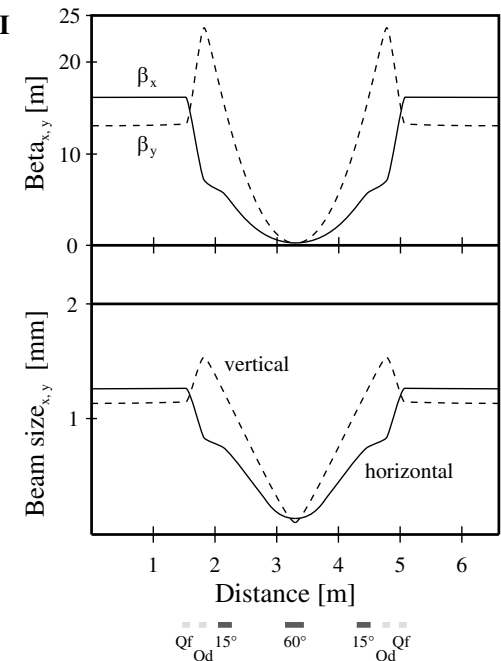

II

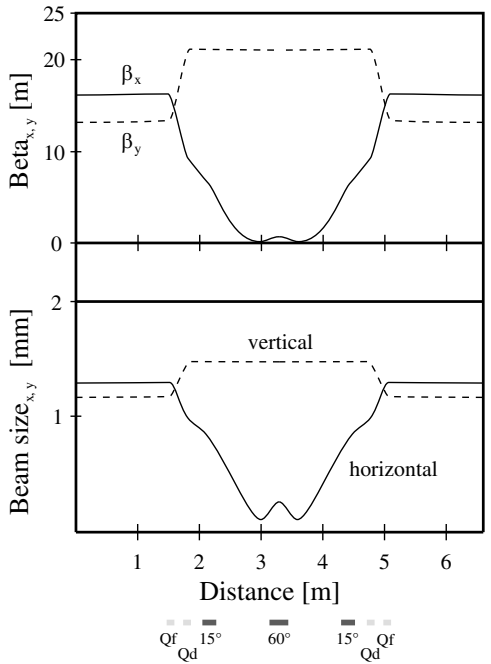

IV

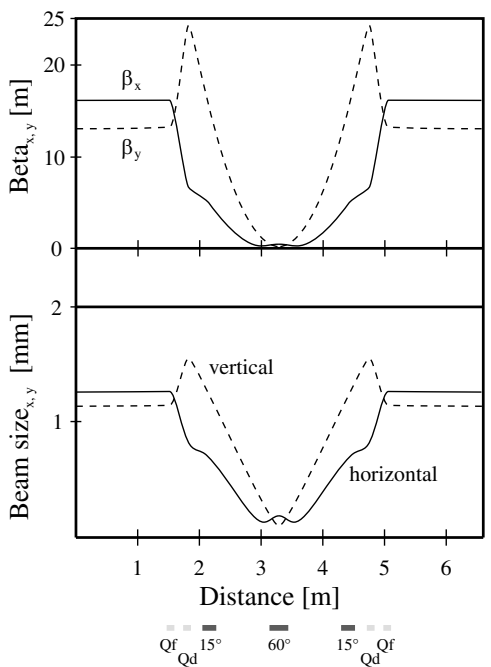

FIG. 3. Lattice functions and sizes in a quarter ring sections at working points I-IV. A detailed description is in the main text.

All calculations were made for a beam with an emittance of $\epsilon=10 \mathrm{~mm} \mathrm{mrad}$ and an initial momentum spread of $\Delta p / p=1 \times 10^{-3}$. The necessary quadrupole strengths at working point $\mathrm{I}$ are $k_{x}=7.2 \mathrm{~m}^{-2}$ and $k_{y}=$ $-6.3 \mathrm{~m}^{-2}$, with chromaticities $\xi_{x}=-2.56$ and $\xi_{y}=$ -0.97 .

At point II, the corresponding quadrupole strengths are $k_{x}=10.2 \mathrm{~m}^{-2}$ and $k_{y}=-8.4 \mathrm{~m}^{-2}$, with chromaticities $\xi_{x}=-3.67$ and $\xi_{y}=-1.8$.

A beam waist in the middle of the $60^{\circ}$ deflector is achieved only at working points III and IV, where $k_{x}=14.5 \mathrm{~m}^{-2}$ and $k_{y}=-18.4 \mathrm{~m}^{-2}$, with chromaticities $\xi_{x}=-5.19$ and $\xi_{y}=-13.5$ for the former, and $k_{x}=$ $15.2 \mathrm{~m}^{-2}$ and $k_{y}=-18.8 \mathrm{~m}^{-2}$, with chromaticities $\xi_{x}=$ -5.98 and $\xi_{y}=-14.0$ for the latter.

The calculated tune values are displayed in a tune diagram where resonances up to third order are shown; see Fig. 4.

\section{OPTICAL ELEMENTS}

As mentioned in the last section, the ring consists of separated function bending and focusing elements. The lattice was kept as simple as possible in order to guarantee easy operation. In comparison to earlier designs [13], a number of parameters had to be changed taking into account the requirements of envisaged experiments.

\section{A. $15^{\circ}$ parallel plate deflector}

Injection as well as part of the $90^{\circ}$ bending in the corner section will be realized with a parallel plate deflector as shown in Fig. 5.

The ions follow a parabolic trace inside this element with the design particle entering in the middle of the deflector. Voltages of $\pm 6.7 \mathrm{kV}$ have to be applied on the electrodes, which are separated by a distance of $10 \mathrm{~cm}$. In order to reduce the disturbing effects of fringe fields [14] 


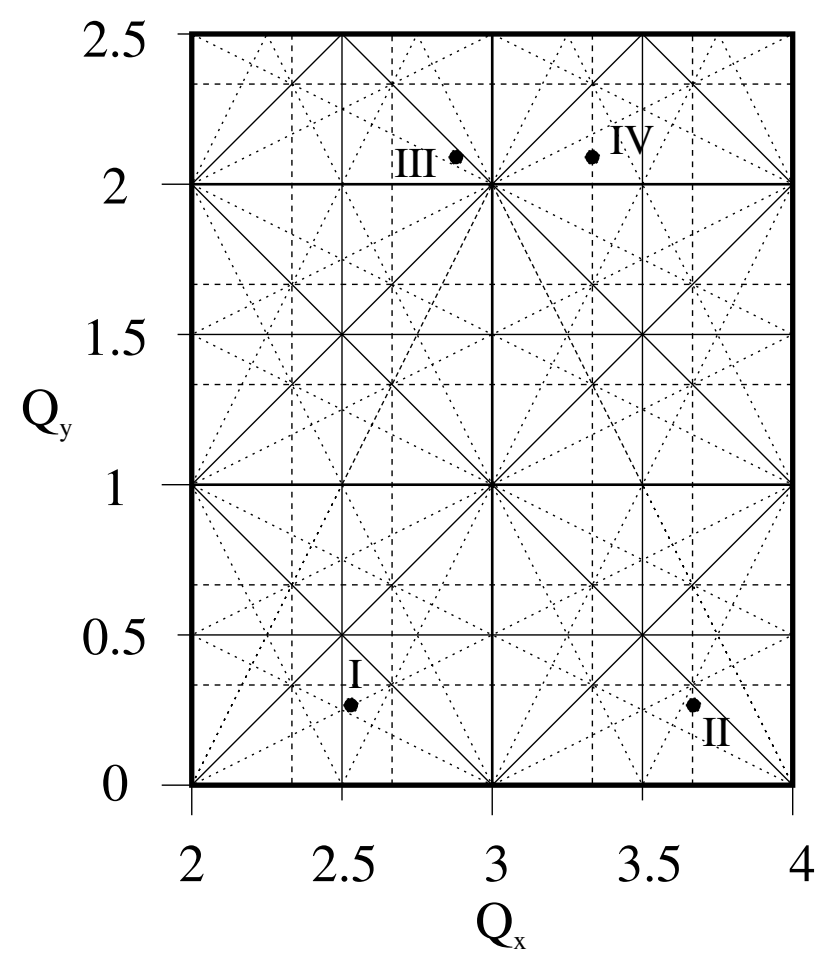

FIG. 4. Tune values in horizontal $\left(Q_{x}\right)$ and vertical $\left(Q_{y}\right)$ directions at working points I-IV

to a minimum, the electrodes were designed with a length and height of $200 \mathrm{~mm}$. This leads to a field inhomogeneity in the central region of the element of less than $1 \times 10^{-4}$.

In case of fragmentation processes especially of heavy organic molecules, one is interested in detecting all par-

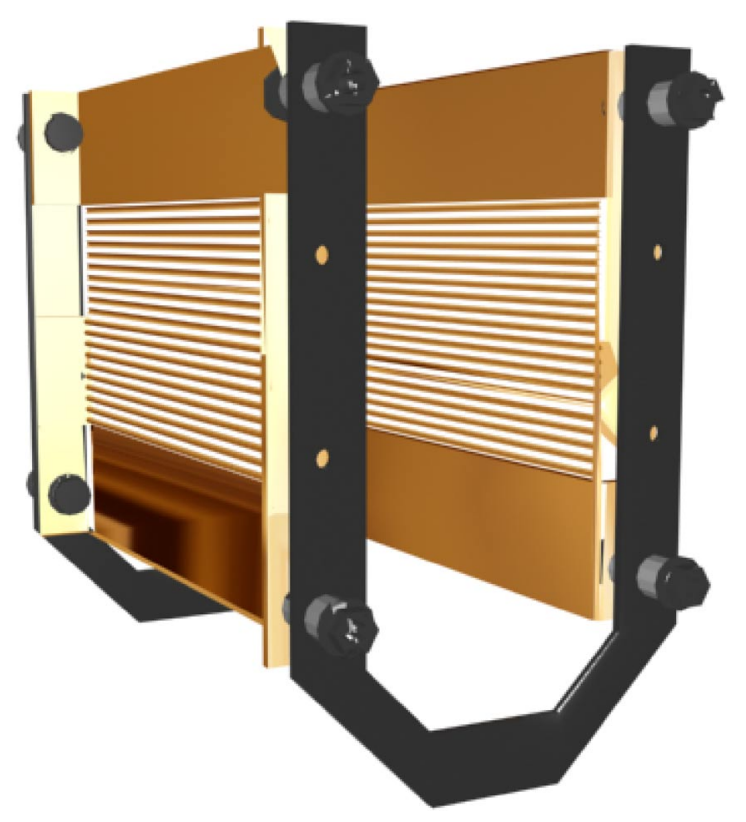

FIG. 5. (Color) $15^{\circ}$ electrostatic parallel plate deflector with central mesh for the detection of light charged fragments. ticles created during the reaction. Especially if light ions like protons are created from an initial heavy molecule, the deflection in the parallel plate deflector is much larger than the original design angle. In case solid electrodes were used, these particles would hit the plates and get lost.

In contrast to existing storage rings, a central $80 \mathrm{~mm}$ mesh was introduced into both plates. This allows detection of the light fragments behind the plates. In contrast to layouts with gaps and correction electrodes, no additional feedthroughs are necessary and the original field structure can be maintained, as shown in Fig. 6.

The support of the electrodes at the same time acts as a grounded shield and reduces the fringe fields in the entrance and exit region considerably.

\section{B. $60^{\circ}$ cylinder deflector}

The main bending in the storage ring is done in $60^{\circ}$ cylinder deflectors placed in a pillbox-type cavity, Fig. 7. In contrast to the parallel plate deflectors, these elements are limited to a specific bending angle by their geometrical dimensions. Again, grounded shields at the entrance

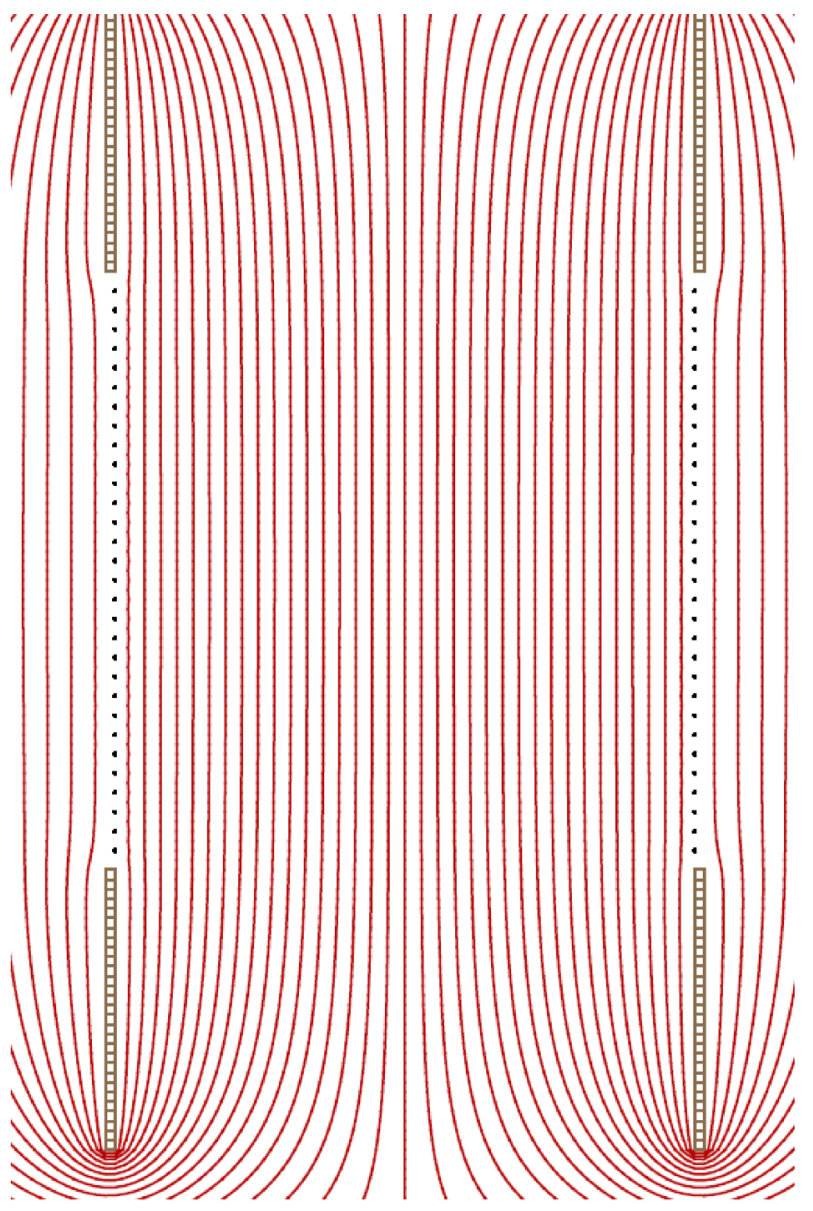

FIG. 6. (Color) Calculated potential distribution within the electrostatic $15^{\circ}$ deflector. 


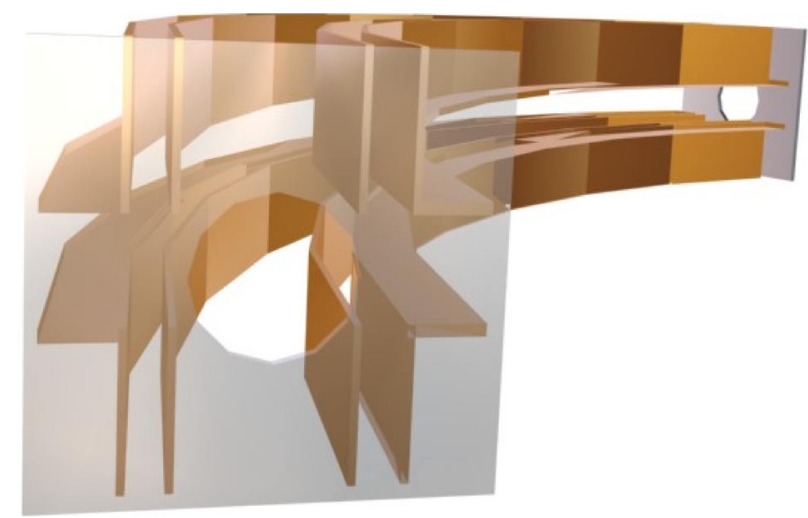

FIG. 7. (Color) Electrostatic cylinder deflector with central gap for detection of charged fragments with small mass differences compared to the original beam.

and exist of this element limit the fringe field effects on the circulating beam.

Experimental requirements had a large impact on the final layout of the element. If one assumes that the longitudinal velocities of all particles involved in fragmentation experiments stays constant and the complete energy release in the order of $10 \mathrm{eV}$ from, e.g., Coulomb explosion is transferred into transverse motion of the fragments, one can calculate a cone in which all fragments propagate through the machine. It comes out that heavy fragments at $50 \mathrm{keV}$ beam energy and mass differences $|\Delta m / m|<20 \%$ as compared to the original beam are displaced by only a few $\mathrm{mm}$ and still enter the cylinder deflector.

In simulations with SimIon it was found that, depending on the ion species and the corresponding charged fragments, the latter will eventually hit the deflector electrodes under a small angle and get lost. In order to be able to detect these fragments, a central gap was introduced into both electrodes and additional correction electrodes were placed to compensate the field disturbances.

While the field in the central region of the element is identical to the field inside a conventional deflector with solid plates, the charged fragments can leave the element and be detected in the outside region.

A major concern was the unavoidable coupling in this element between transverse and longitudinal phase space. This effect leads to a natural longitudinal dispersion and quickly destroys any time structure of the beam. In order to find out how large these defocusing effects are, tracking simulations were done, taking into account the fringe fields of all elements.

The minimum extension in the longitudinal direction can be found if one assumes an initially perfect beam with zero length. Forty particles where placed inside an initial beam radius of $5 \mathrm{~mm}$. After one revolution, the length of the bunch is $\pm 2 \mathrm{~mm}$, which corresponds to a

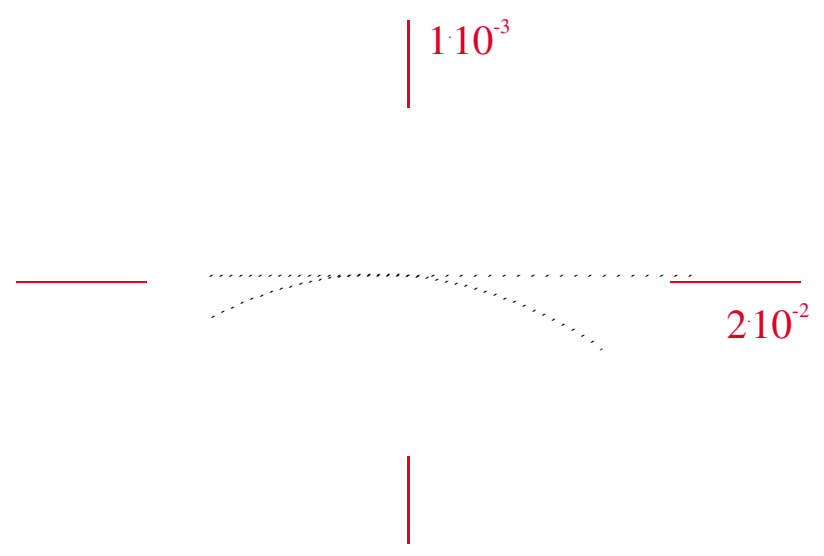

FIG. 8. (Color) Minimum longitudinal dispersion in the ring of FIRE after one revolution in the machine.

time structure of $0.5 \mathrm{~ns}$, Fig. 8 . A dispersion of this size can be counteracted with the integrated rebuncher. However, intrabeam scattering also leads to longitudinal defocusing of the beam and is very strong at these low energies. Theoretical models can hardly predict the amount of additional dispersion caused by this ion interaction. Therefore systematic measurements on the intrabeam scattering rates at low energies will be carried out this year at the TSR in Heidelberg.

\section{Quadrupole doublets}

The transverse dimensions of the circulating beam are controlled by electrostatic quadrupole doublets. During operation, it is important that all of the fields in the machine can be adjusted independently.

Figure 9 shows the calculated field gradient at $1 \mathrm{~cm}$ distance from the central orbit in an electrostatic quadrupole doublet. While the voltages in the first quadrupole are kept at $3 \mathrm{kV}$ all the time, the voltages in the second lens start at $3 \mathrm{kV}$ and are increased by steps of $200 \mathrm{~V}$. This unequal voltage distribution corresponds to the different focusing strengths of the element depending on the working point chosen.

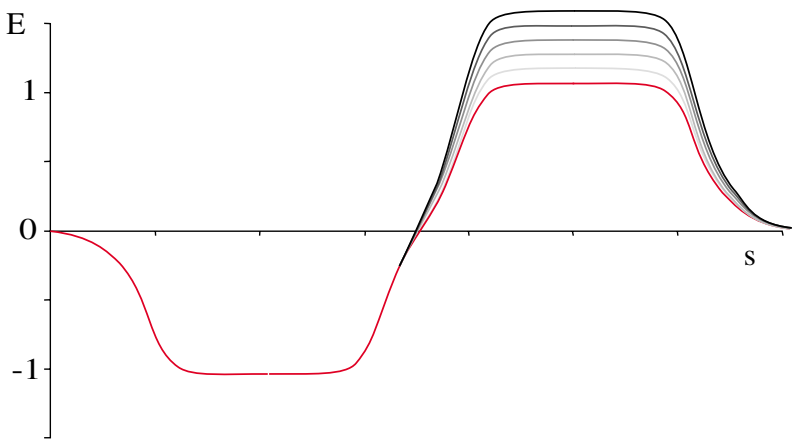

FIG. 9. (Color) Potential distribution inside the electrostatic quadrupole doublet; arbitrary units. 


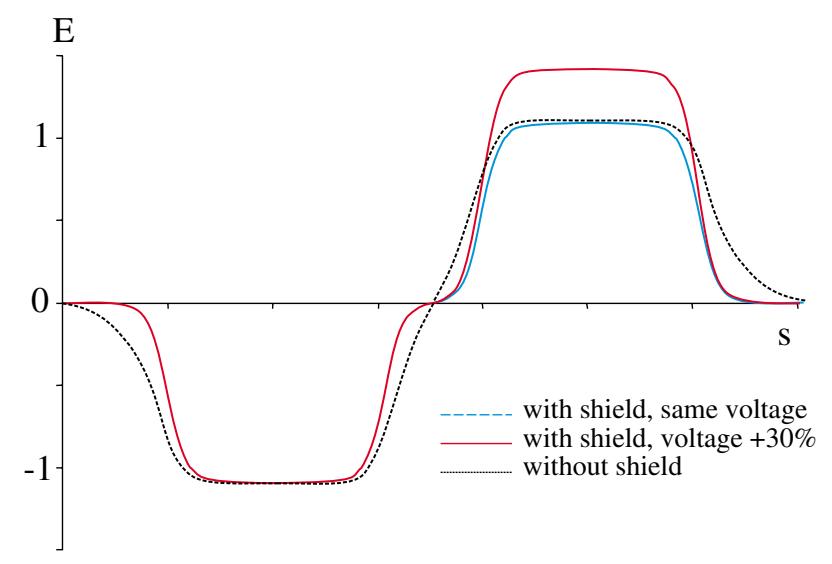

FIG. 10. (Color) Comparison of the potential distribution inside the electrostatic quadrupole doublet with and without grounded shields between the lenses; arbitrary units.

It can clearly be seen that the intersection with the $x$ axis and thus the effective length of both lenses changes with an increasing difference in the voltages. This coupling between the two focusing elements leads to unwanted effects during the operation of the storage ring. Changing the voltage in one quadrupole would automatically modify the second lens as well.

To counteract this coupling, grounded shields were installed between the electrodes at a distance of $10 \mathrm{~mm}$. Figure 10 shows the direct comparison between the calculated fields with and without additional shields. The fringe fields can clearly be reduced and-more importantly-the coupling between the two quadrupoles vanishes.

In existing electrostatic storage rings, closed orbit correction is done with additional steerers placed closed to the corner sections of the machine. If one aims for detecting all the particles created in a fragmentation process, these elements avoid placing the detectors in the region. For that reason and for a clear decoupling of the quadrupole lenses, a vertical steerer was introduced, as shown in Fig. 11.

The distance between the two quadrupoles was extended to $150 \mathrm{~mm}$, which completely decouples the fields of the elements. In case the beam is disturbed by, e.g.,

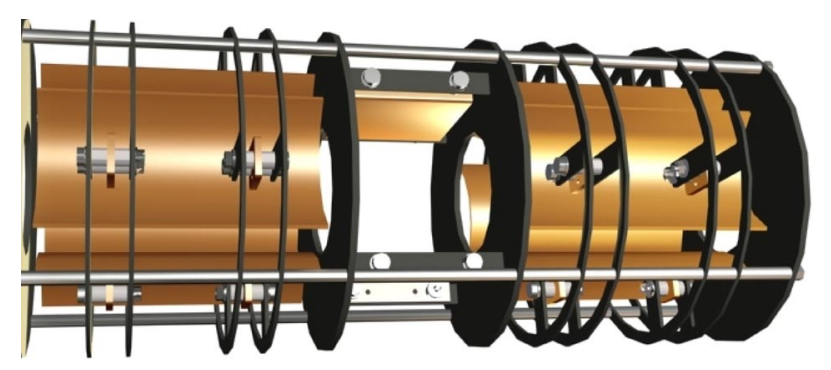

FIG. 11. (Color) Electrostatic quadrupole doublet with integrated steerer.
TABLE I. Summary of design parameters.

\begin{tabular}{lc}
\hline \hline General parameters & \\
Maximum energy & $50 \mathrm{keV}$ \\
Circumference & $25.45 \mathrm{~m}$ \\
Revolution time & $9.9 \mu \mathrm{s}(\mathrm{p})$ \\
$15^{\circ}$ deflectors & \\
Plate area & $200 \mathrm{~mm} \times 200 \mathrm{~mm}$ \\
Plate distance & $100 \mathrm{~mm}$ \\
Mesh height & $80 \mathrm{~mm}$ \\
Voltage & $\pm 6.7 \mathrm{kV}$ \\
$60^{\circ}$ deflectors & \\
Height & $100 \mathrm{~mm}$ \\
Radii & $235 \mathrm{and} 265 \mathrm{~mm}$ \\
Shield distance & $10 \mathrm{~mm}$ \\
Voltage & $\pm 6 \mathrm{kV}$ \\
Quadrupoles & \\
Length & $100 \mathrm{~mm}$ \\
Distance between lenses & $90 \mathrm{~mm}$ \\
Aperture radius & $25 \mathrm{~mm}$ \\
Shield distance & $10 \mathrm{~mm}$ \\
Voltage & $\pm 3 \mathrm{kV}$ \\
Steerer length & $50 \mathrm{~mm}$ \\
Steerer plate distance & $60 \mathrm{~mm}$ \\
\hline
\end{tabular}

field errors, small voltages can be applied on the vertical steerer to counteract beam shifts. The horizontal steering is achieved by the parallel plate deflectors, where small voltages can be added, if necessary.

The electric and mechanic parameters of the machine and its components are summarized in Table I.

\section{CONCLUSION}

The electrostatic storage ring of the Frankfurt Ion Storage Experiments will cover a wide range of possible fields and serve as a true multipurpose, multiuser facility. Because of the mass independence of the electrostatic rigidity of all optical elements, all ions from light protons to heavy biomolecules can be stored in the machine.

An integrated reaction microscope allows the study of many-particle dynamics with unrivaled resolution and completeness. It will be possible to analyze the kinematics of many-particle processes with highest precision.

In comparison to earlier design studies, major changes in the layout of the optical elements were made, taking into account the requirements of envisaged experiments.

As the central machine of the new Stern-Gerlach Center at Frankfurt University, the electrostatic storage ring will combine the efforts of the institutes of nuclear and applied physics, biophysics, and physical chemistry and allow novel views into the dynamics of complex organic matter. 


\section{ACKNOWLEDGMENTS}

The authors thank Søren Pape Møller for his valuable support during the design process. Furthermore, the help of the Studienstiftung des deutschen Volkes is truly appreciated.

[1] S. P. Møller, in Proceedings of the European Particle Accelerator Conference, Stockholm, Sweden, 1998 (Institute of Physics, London, 1998).

[2] T. Tanabe et al., Nucl. Instrum. Methods Phys. Res., Sect. A 482, 595 (2002).

[3] T. Tanabe and K. Noda, Nucl. Instrum. Methods Phys. Res., Sect. A 496, 233 (2003).

[4] T. Tanabe, K. Noda, M. Saito, S. Lee, Y. Ito, and H. Takagi, Phys. Rev. Lett. 90, 193201 (2003).

[5] S. B. Nielsen et al., Phys. Rev. Lett. 87, 228102 (2001).

[6] J. Ullrich et al., Rep. Prog. Phys. 66, 1463 (2003).
[7] T. Tanabe, K. Noda, and E. Syresin, in Proceedings of COOL03, Mt. Fuji, Japan, 2003 [Nucl. Instrum. Methods Phys. Res., Sect. A (to be published)].

[8] J. U. Andersen et al., Rev. Sci. Instrum. 73, 1284 (2002).

[9] H. Schmidt-Böcking et al., Europhysics News 33, 210 (2002), www.europhysicsnews.com.

[10] R. von Hahn et al., in Proceedings of the European Accelerator Conference, Lucerne, Switzerland, 2004 (to be published).

[11] DESIREE project at MSL, http://www.msi.se/futureproj/ desiree.pdf

[12] C. P. Welsch et al., in Proceedings of the European Accelerator Conference, Lucerne, Switzerland, 2004 (Ref. [10]).

[13] C.P. Welsch et al., in Proceedings of the Particle Accelerator Conference, Portland, OR, 2003 (IEEE, Piscataway, NJ, 2003).

[14] C. P. Welsch et al., in Proceedings of the European Particle Accelerator Conference, Paris, 2002 (EPSIGA and CERN, Geneva, 2002). 